\title{
INFECTIOUS COMPLICATIONS AS A PREDICTOR OF MORTALITY IN PATIENTS WITH NON-HODGKIN LYMPHOMA RECEIVING RITUXIMAB- CONTAINING CHEMOTHERAPY
}

\author{
Patricia Meza-Meneses ${ }^{1}$, Patricia Cornejo-Juárez ${ }^{2}$, Diana Vilar-Compte ${ }^{2}$ \\ ANd PATRICIA Volkow-Fernández ${ }^{2 *}$ \\ ${ }^{1}$ Department of Infectious Diseases, Hospital Regional de Alta Especialidad de Ixtapaluca, Edo. de México; \\ 2Instituto Nacional de Cancerología, Mexico City, Mexico.
}

\begin{abstract}
Background: Rituximab is a monoclonal antibody that increases the disease-free and overall survival of patients with nonHodgkin lymphoma (NHL) CD20+. The objective of this study is to describe the prevalence and spectrum of infections in patients with $\mathrm{NHL}$ receiving rituximab-containing chemotherapy and the impact on survival. Materials and Methods: From January 2011 to December 2012, all patients diagnosed with NHL who received at least one dose of rituximab were included. Results: During the study period, 265 patients received rituximab; 108 (40.8\%) males; the mean age was $60 \pm 15$ years. There were 177 infections in 85 patients, being the most common febrile neutropenia $(n=38 ; 21.5 \%)$ and mucosal barrier injury-related infections $(n=28 ; 15.8 \%)$. In 88 events ( $49 \%)$, there was a microbiologic diagnosis, being bacterial infection the most frequent (39.6\%), but tuberculosis (TB) was developed in 4 cases (1.5\%; incidence rate 721/100,000 person-year). During follow-up, 71 patients died (27\%); in 35 cases, it was related to infection. There were no differences in follow-up between those who died due to infection versus those who died from another cause $(p=0.188)$. Multivariate analysis for mortality showed that age $>60$ years, failure to achieve a complete response, and development of an infectious complication increased the risk of death. Conclusions: It is important to perform a screening test for TB in all patients who will receive rituximab and maintain a constant monitoring to detect an infectious process and begin treatment as soon as possible. (REV INVEST CLIN. 2019;71:275-82)
\end{abstract}

Key words: Rituximab. Non-Hodgkin lymphoma. Infection. Tuberculosis.

Corresponding author:

*Patricia Volkow-Fernández

Department of Infectious Diseases

Instituto Nacional de Cancerología

Av. San Fernando, No. 22

Del. Tlalpan

Received for publication: 06-02-2019

C.P. 14080 , Mexico City, Mexico

E-mail:pvolkowf@gmail.com

Approved for publication: 03-04-2019

DOI: $10.24875 / R I C .19002982$ 


\section{INTRODUCTION}

Rituximab is a monoclonal antibody against the CD20 protein present on the surface of $B$ lymphocytes. It has demonstrated to increase the disease-free and overall survival (OS) of patients with non-Hodgkin lymphoma $(\mathrm{NHL}) \mathrm{CD} 20+{ }^{1}$.

Previous reviews studying the infections associated with rituximab in patients with lymphoma, the addition of this drug to other chemotherapeutic agents did not result in an increased incidence of infections after more than 10 years of use; however, when used alone for maintenance for follicular lymphoma or in special populations (e.g., HIV-infected patients), rituximab comprised an additive risk factor for infections ${ }^{2}$. However, rituximab in combination with chemotherapy exerts cytotoxic effects on T cells that can lead to hypogammaglobulinemia and late-onset neutropenia, which have been related to higher risk of infection ${ }^{2}$. An increased risk has been reported for hepatitis B virus (HBV) and Hepatitis $C$ virus reactivation ${ }^{1}$. Furthermore, a higher frequency of progressive multifocal leukoencephalopathy (PML) has been reported ${ }^{2}$. Other infections with a possibly increased occurrence associated are Pneumocystis jirovecii pneumonia; enterovirus encephalitis; Parvovirus B19; Cytomegalovirus; West Nile virus; parasitic infections, and mycobacterial infections ${ }^{2}$.

The incidence of infectious complications and the outcome of these events in rituximab-treated patients with $\mathrm{NHL}$ have not been acknowledged in middle-income countries, where there is a higher prevalence of tuberculosis (TB) and fungal infections (e.g., histoplasmosis). Thus, the spectrum of infections in patients in these countries will be expected to differ from that reported in literature on high-income countries. The aim of the study was to describe the incidence, spectra, infectious complications, and outcome in patients with $\mathrm{NHL}$ receiving rituximab-containing chemotherapy regimens. This is a retrospective study that included all patients from January 1, 2011, to December 31, 2012, who were diagnosed with $\mathrm{NHL}$ at the National Cancer Institute (INCan) who received at least one dose of rituximab.

\section{MATERIALS AND METHODS}

The INCan is a 135-bed referral teaching hospital located in Mexico City for adult patients with cancer, with an average of 170,000 medical visits, 7500 hospital discharges, and 3500 major surgical procedures per year.

This is a retrospective study that included all patients from January 1, 2011, to December 31, 2012, who were diagnosed with $\mathrm{NHL}$ and who received at least one dose of rituximab. The Government's program "popular insurance," included patients with diffuse large B-cell non-Hodgkin's lymphoma since 2011, and implemented standard chemotherapy plus rituximab at no cost, which is why almost all patients receive this combination. Patients with HIV infection were excluded from the study. The study was reviewed and approved by the Institutional Review Board (INCAN/CI/212/14).

Demographics, comorbidities, lymphoma characteristics (type, stage), chemotherapy, and number of doses of rituximab received were recorded. Patients were followed up from the first dose of rituximab received, up to 24 months after the last course of rituximab, or death.

An infectious episode was diagnosed when there was a microbiology-documented infection, radiographic or histological signs suggestive of an infectious process, or febrile neutropenia. Only infections Grades 3-5 of the common terminology criteria for adverse events were analyzed ${ }^{3}$. Absolute neutrophil and lymphocyte count, length of hospital stay, microbiological isolations, and outcome were registered for each infectious episode and at the end of the follow-up.

Main outcome was response to chemotherapy, classified as complete response (CR), partial response, progression, relapse, or death. Patients were considered lost to follow-up $\geq 6$ months after their past medical visit. Positron emission tomography with 18-fluoro-D-glucose integrated with computed tomography (CT) is performed at lymphoma diagnosis and after finishing treatment ( 8 weeks after the last chemotherapy). At the middle of the number of cycles (third/sixth or fourth/eight), a CT-scan is performed. The number of chemotherapy cycles is considered in the base of the lymphoma stage: 6 for early and 8 for advanced.

\section{Definitions:}

Clinically documented infection: fever accompanied by clinical findings. 
Mucosal barrier injury - bloodstream infection (MBI$\mathrm{BSI}$ ): patients who received chemotherapy with severe neutropenia (neutrophils $<500$ cells $/ \mathrm{mm}^{3}$ at least in two occasions or $<100$ cells $/ \mathrm{mm}^{3}$ at least on one occasion), or an allogeneic hematopoietic stem-cell transplantation, or Grade 3-4 gastrointestinal graft versus host disease, or diarrhea $\geq 1 \mathrm{~L}$, or Grade 3-4 mucositis, and at least one blood specimen identified by culture with intestinal organisms ${ }^{4}$.

Pneumonia: radiological criteria on chest $\mathrm{X}$-ray or a CT scan plus one or more of the following: fever $\left(\geq 38^{\circ} \mathrm{C}\right)$ or hypothermia $\left(<35^{\circ} \mathrm{C}\right)$; new cough with or without sputum production; pleurisy chest pain; dyspnea, and altered breathing sounds on auscultation.

Central-related bloodstream infection (CRBSI) was considered if time to positivity between blood cultures taken from the catheter was two or more hours apart from the blood culture taken from peripheral puncture, with signs of systemic infection (fever, chills, and/or hypotension), and no apparent source of infection except the catheter, and/or catheter-tip culture positive for the same organism (when the catheter was removed), and/or signs and symptoms of catheter entry-site infection ${ }^{5}$.

Neutropenic colitis required the presence of an acute abdomen with $\mathrm{CT}$ findings consistent with intestinal inflammation.

Urinary tract infection was defined as the coincidence of fever, typical symptoms, significant bacteriuria, and positive urine culture with an infectious pathogen.

\section{Statistical analysis}

Student t-test or Mann-Whitney U-test was used to compare continuous variables, and Chi-square or Fisher exact test was employed to compare categorical variables. A logistic regression analysis was performed for predicting risk for infection; variables with $p<0.5$ on univariate analysis were included in multivariate analysis. Odds ratios with $95 \%$ confidence intervals were calculated. Overall Survival (OS) rates were estimated by the Kaplan-Meier method and the log-rank test. $p \leq 0.05$ was considered statistically significant. Data were analyzed using STATA ver. 14 statistical software (Stata Corp., College Station, TX, USA).

\section{RESULTS}

During the study period, 265 patients were included: 108 (40.8\%) men; mean age was $60 \pm 15$ years; 46 (17\%) had diabetes mellitus (DM). An aggressive type of lymphoma (diffuse large B-cell $-\mathrm{n}=205$, mantle-cell $-n=4$, Burkitt $-n=1$, or marginal-zone $-n=1$ ) was documented in 211 patients (79.6\%); 180 (67.9\%) were in Stage 3 or 4 (Ann Arbor staging system), and 261 (98.5\%) received standard first-line treatment as follows: rituximab plus cyclophosphamide, doxorubicin, vincristine, and prednisone (R-CHOP). Other clinical characteristics are presented in Table 1.

Eighty-five patients (32\%) developed a Grade 3-5 infection; incidence rate was 41.98 infections per 100,000 person-days. There were 177 events in the 85 patients, and 42 (49\%) of these had more than one event, (median, 2 events/person; interquartile range [IQR], 1-3 events). There were significantly more patients with an infection that did not achieve CR with the first chemotherapy scheme $(n=31,36.5 \%)$ versus those without infection ( $n=25,13.9 \%$; $<<0.001)$. There were no other significant differences in baseline characteristics between patients who developed an infection versus those who did not develop one (Table 1).

Median time to infection since day 1 of therapy was 133 days (IQR, 53-270 days). Most common infections comprised febrile neutropenia $(n=38 ; 21.5 \%)$; MBI-related infections ( $n=28 ; 15.8 \%)$, and pneumonia $(n=38 ; 21.5 \%)$. Other infections and outcomes are depicted in Table 2.

In 88 of the events ( $49 \%$ ), there was a microbiologic diagnosis; the most frequent were bacteria $(n=73$; 41.2\%) (Fig. 1). 10 patients (5.6\%) had a virus (five varicella-zoster, one coronavirus, one metapneumovirus, one influenza virus, and one papillomavirus); six (3.39\%) had fungi (four Candida spp. and two Aspergillus spp.); four (1.5\%) had Mycobacterium tuberculosis complex (incidence rate $721 / 100,000$ personyear), and one patient had a parasite (Strongyloides stercoralis). In 86 cases (48.6\%), there was not a microorganism identified: 38 cases (21.5\%) with fever and neutropenia, 16 (9\%) with $\mathrm{MBI}, 14$ (7.9\%) with pneumonia, 5 (2.8\%) with skin and soft tissue infection, 6 (3.4\%) with abdominal sepsis, 2 (1.1\%) with gastroenteritis, $1(0.6 \%)$ with encephalitis, and $4(2.3 \%)$ with other infections. 
Table 1. Demographic and clinical characteristics in patients with non-Hodgkin lymphoma who received rituximab in comparison with patients who developed an infection versus those who did not

\begin{tabular}{|c|c|c|c|c|}
\hline $\begin{array}{l}\text { Characteristics } \\
\text { n }(\%)\end{array}$ & $\begin{array}{l}\text { Non-infection } \\
n=180(67.9)\end{array}$ & $\begin{array}{c}\text { Infection } \\
\mathrm{n}=85(32.1)\end{array}$ & $\begin{array}{c}\text { Total } \\
n=265\end{array}$ & $\mathrm{p}$ \\
\hline Male patients & $71(39)$ & $37(44)$ & $108(40.8)$ & 0.528 \\
\hline Age* (years) & $60.8 \pm 15$ & $60.3 \pm 15$ & $60.5 \pm 15$ & 0.961 \\
\hline Above 60 years of age & $87(48)$ & $45(53)$ & $132(50)$ & 0.484 \\
\hline Diabetes mellitus & $31(17)$ & $15(17)$ & $46(17)$ & 0.932 \\
\hline Other cancers ${ }^{\alpha}$ & $14(8)$ & $4(5)$ & $18(7)$ & 0.354 \\
\hline Follow-up* (months) & $28.6 \pm 11.9$ & $18.9 \pm 13.3$ & $25.5 \pm 13.1$ & $<0.001$ \\
\hline Lymphoma type & & & & 0.158 \\
\hline Follicular & $41(22.8)$ & $13(15.3)$ & $54(20.3)$ & \\
\hline Diffuse large B-cell & $134(74.4)$ & $71(83.5)$ & $205(77.4)$ & \\
\hline Mantle cell & $3(1.6)$ & $1(1.2)$ & $4(1.5)$ & \\
\hline Burkitt & $1(0.6)$ & 0 & $1(0.4)$ & \\
\hline Marginal zone & $1(0.6)$ & 0 & $1(0.4)$ & \\
\hline Stage ${ }^{\S}$ & & & & 0.04 \\
\hline I-II & $62(34.4)$ & $19(22.3)$ & $81(30.6)$ & \\
\hline III-IV & $118(65.6)$ & $66(77.7)$ & $184(69.4)$ & \\
\hline First-line CT & & & & 0.760 \\
\hline $\mathrm{CHOP} / \mathrm{COP}$ & $177(98)$ & $84(99)$ & $261(99)$ & \\
\hline Other & $3(2)$ & $1(1)$ & $4(1)$ & \\
\hline Failure to first CT scheme & $25(13.9)$ & $31(36.5)$ & $56(21.1)$ & $<0.001$ \\
\hline
\end{tabular}

*Median \pm Standard deviation, \&There were 18 patients with another neoplasia: 5 synchronic and 13 non-synchronic (four thyroid carcinomas, five skin neoplasia [three basocellular and two epidermoid], two breast cancers, two cervical cancer, one vulvar, one hepatocellular carcinoma, one myeloid leukemia, one meningioma, and one teratoma). §Stage was described in 258 patients (in seven, the staging was not performed), CT: computed tomography, CHOP: cyclophosphamide, doxorubicin, vincristine, and prednisone; COP: cyclophosphamide, vincristine, and prednisone.

None of the patients were positive for HBV surface antigen $(\mathrm{HBsAg})$ at baseline screening, there were six patients with positive anti-core HBV (anti-HBC) who did not receive pre-emptive antiviral treatment, and none of these patients had HBV reactivation during follow-up.

We did not find any case of histoplasmosis, despite the fact that Mexico has a high prevalence of this infection. Probably there is an underestimation, due to the fact that blood marrow cultures are not performed as a routine unless there is suspicion of an infection that can be documented by this study.

Median follow since first rituximab dose was $18.9 \pm$ 13.3 months. There were not severe adverse events related to rituximab. During follow-up, 71 patients died ( $27 \%$ ); in 35 cases $(49.3 \%)$, the cause of death was infection: bacteria were isolated in 17 cases
(48.6\%), fungi in two (5.7\%), virus in two (5.7\%), and in 14 cases ( $40 \%)$, there was not a microorganism identified. The most frequent death-related infections were pneumonia $(n=14 ; 40 \%), M B I-B S I(n=7 ; 20 \%)$, and skin/soft tissue infections ( $n=5 ; 14 \%$ ) (Table 2). Mortality between patients who developed an infection event and those who did not is shown in figure 2. Of all the patients who died, $65(91.5 \%)$ were in progression or relapse, compared with 21 (10.8\%) of those who survived $(p<0.0001)$. There were no differences when compared those patients with progression or relapse who died related with an infection ( $n$ $=33,94.3 \%$ ) compared with those who died due to another cause $(n=32,88.9 \% ; p=0.673)$.

Multivariate analysis for mortality revealed that age $>60$ years, failure to achieve $C R$, and development of an infectious complication increased the risk of death (Table 3). 
Table 2. Frequency of infectious events and outcome in patients with non-Hodgkin lymphoma who received rituximab

\begin{tabular}{lcc}
\hline $\begin{array}{l}\text { Infection } \\
\mathrm{n}(\%)\end{array}$ & $\begin{array}{c}\text { Total } \\
(\mathrm{n}=177)\end{array}$ & $\begin{array}{c}\text { Death related with infections } \\
(\mathrm{n}=35)\end{array}$ \\
\hline Pneumonia & $38(21.5)$ & $14(40)$ \\
Febrile neutropenia & $38(21.5)$ & 0 \\
Mucosal barrier injury BSI* & $28(15.8)$ & $1(20)$ \\
Urinary tract infection & $19(10.7)$ & $5(14.2)$ \\
Skin and soft tissue infection & $17(9.6)$ & $3(8.5)$ \\
Abdominal sepsis & $12(6.8)$ & $3(8.5)$ \\
CRBSI\& & $16(9)$ & 0 \\
Meningitis & $2(1.1)$ & $2(5.7)$ \\
Other & $7(4)$ &
\end{tabular}

*BSI: bloodstream infection, ${ }^{\star} \mathrm{CRBSI}$ : catheter-related bloodstream infection.

Figure 1. Microbial characteristic of bacteria isolated from patients with non-Hodgkin lymphoma who received rituximab $(n=70)$.

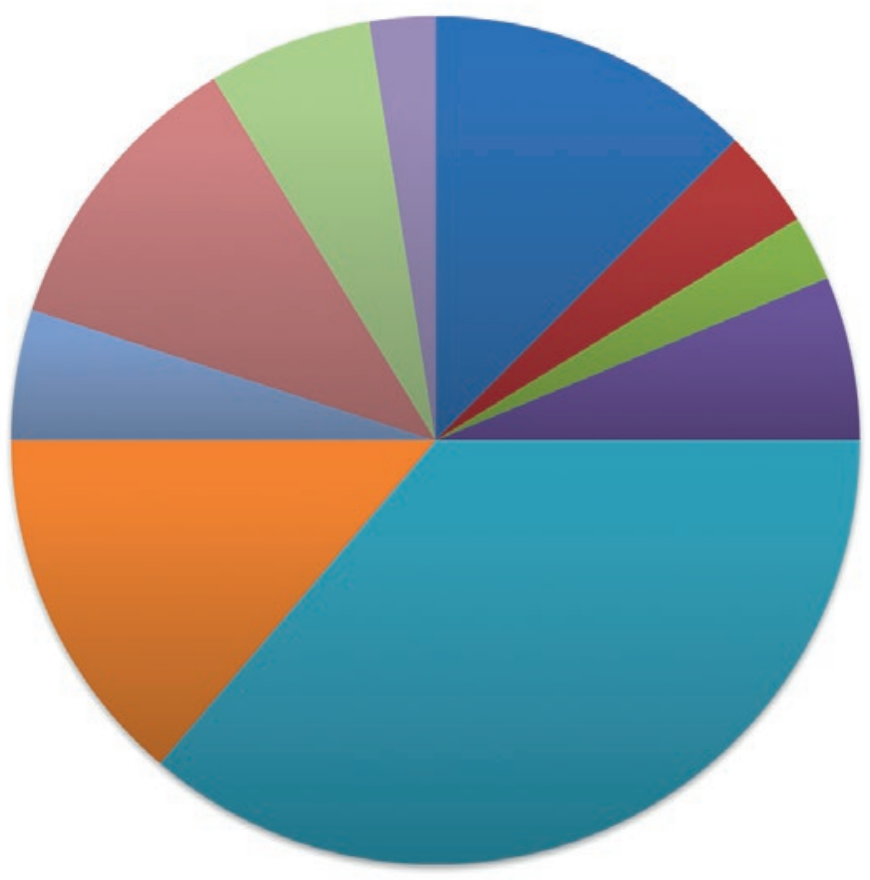

Coagulase-Negative
Staphylococci
Methicilin-susceptible
Staphylococcus aureus
Vancomycin-Resistant
Enterococci
Streptococcus spp
Escherichia coli
Klebsiella spp
Enterobacteriacea
= Pseudomonas aeruginosa

\section{DISCUSSION}

In this study, we report 177 infections in 85 patients $\mathrm{NHL}$ who received rituximab and were followed for a mean of 18.9 months. Only in $50 \%$ of the cases, there was a microbiology etiology, being bacteria the most frequent cause (39.6\%) followed by virus (5.6\%) and TB (2.3\%).
In a recent report that included 101 patients treated with rituximab (with autoimmune and hematological diseases), $73.3 \%$ presented infectious events ${ }^{6}$. Other studies which have included only patients with hematological diseases, the incidence of serious infections was reported between 50 and 58\%: $11 \%$ were opportunistic pathogens and $13 \%$ were fatal episodes ${ }^{6,7}$. These data are similar to the results we found: rate 
Table 3. Multivariate analysis of factors associated with mortality patients with non-Hodgkin lymphoma who received rituximab

\begin{tabular}{|c|c|c|c|c|c|c|}
\hline \multicolumn{7}{|l|}{ Risk of death } \\
\hline \multirow[t]{2}{*}{ Characteristic } & \multirow{2}{*}{$\begin{array}{c}\text { Alive } n=194 \\
n(\%)\end{array}$} & \multirow{2}{*}{$\begin{array}{c}\text { Death } n=54 \\
n(\%)\end{array}$} & \multicolumn{2}{|c|}{ Univariate } & \multicolumn{2}{|c|}{ Multivariate } \\
\hline & & & OR $(95 \% \mathrm{Cl})$ & $\mathrm{p}$ & OR $(95 \% \mathrm{Cl})$ & $\mathrm{p}$ \\
\hline \multicolumn{7}{|l|}{ Age (year) } \\
\hline$<60$ & $110(57)$ & $16(30)$ & $\begin{array}{c}3.1 \\
(1.6-5.9)\end{array}$ & $<0.001$ & $\begin{array}{c}1.00 \\
3.7(1.7-8.2)\end{array}$ & 0.001 \\
\hline$>60$ & $84(43)$ & $38(70)$ & & & & \\
\hline \multicolumn{7}{|c|}{ Achieve complete response } \\
\hline Yes & $173(89)$ & $4(7)$ & $\begin{array}{c}102.9 \\
(33.7-313.9)\end{array}$ & $<0.001$ & $\begin{array}{c}1.00 \\
3.9(1.8-8.8)\end{array}$ & 0.001 \\
\hline No & $21(11)$ & $50(93)$ & & & & \\
\hline \multicolumn{7}{|c|}{ Infectious complication } \\
\hline No & $153(79)$ & $16(30)$ & $8.8(4.5-18.6)$ & $<0.001$ & $\begin{array}{ll} & 1.00 \\
7.9 & (3.8-17)\end{array}$ & $<0.001$ \\
\hline Yes & $41(21)$ & $38(70)$ & & & & \\
\hline
\end{tabular}

$\mathrm{Cl}$ : confidence interval, OR: odds ratios.

of serious infection was $66.8 \%$, incidence rate for opportunistic pathogens $6.8 \%$, and for the fatality rate associated with infection $13.2 \%$. The lower percentage of opportunistic infections that we found could be explained as due to the lack of molecular tests to identify non-cultivable or difficult-to-grow pathogens at our center.

Considering the etiology of infections reported in patients with lymphoid malignancies treated with rituximab have been described: $31 \%$ for bacterial infections, $10 \%$ for viral infections, and $1 \%$ for fungal infections ${ }^{8}$. In a systematic review of 944 patients, of whom 535 received rituximab, overall number of opportunistic infections diagnosed was 299 (31.7\%), $109(36.5 \%)$, of unknown or unspecified origin; 100 (33.4\%), viral reactivations; 44 (14.7\%), bacterial; 26 (8.7\%), fungal; 11 (3.7\%), protozoal, and nine (3\%), viral infections ${ }^{9}$. In the present series, nearly one-half of the episodes were of unknown origin, in $39.6 \%$ a bacterium was identified, in $5.1 \%$ a virus, in $2.3 \%$ a fungus, in $2.3 \%$ a mycobacterium, and in $0.6 \%$, a parasite was identified, without viral reactivations.

Since 2002, an increase of cases of PML has been described as being related to rituximab use ${ }^{10}$. In this report, we did not find any case of PML.
The screening protocol carried out in our hospital for more than 10 years and all patients diagnosed with lymphoma, is to perform serological tests for HIV, $\mathrm{HBV}$, and HCV, since patient admission. Before carrying out this study, we did not have an established protocol for latent TB infection (LTBI). At present, tuberculin test purified protein derivative (PPD) is performed in all $\mathrm{NHL}$ patients who will receive rituximab; and in those with $>5 \mathrm{~mm}$, a CT thorax is done to evaluate if they are candidates to receive isoniazid $300 \mathrm{mg}$ QD for 9 months. Although the majority of individuals infected with $M$. tuberculosis do not develop active disease, they harbor the dormant mycobacterium as a LTBI. The mycobacteria are confined by granuloma through recruitment of CD4 and CD 8 $T$ cells, $B$ cells, and macrophages to the infected site. Rituximab can cause active TB by disrupting the granuloma $^{11}$. Some studies have underestimated the TB prevalence rate associated with different biological therapies ${ }^{11}$. The prevalence found in this group of patients was $1.5 \%$ (721/100,000 incidence rate), considerably higher than TB incidence reported by the World Health Organization for Mexico (21/100,000 population-year) ${ }^{12}$. An important point to consider is the relationship that exists between $\mathrm{TB}$ and $\mathrm{DM}^{13}$, and the high prevalence of DM that exists in Mexican population. However, none of the four patients who 
Figure 2. Kaplan-Meier overall survival curve in patients with non-Hodgkin lymphoma who received rituximab compared with those who developed an infection versus those who did not.

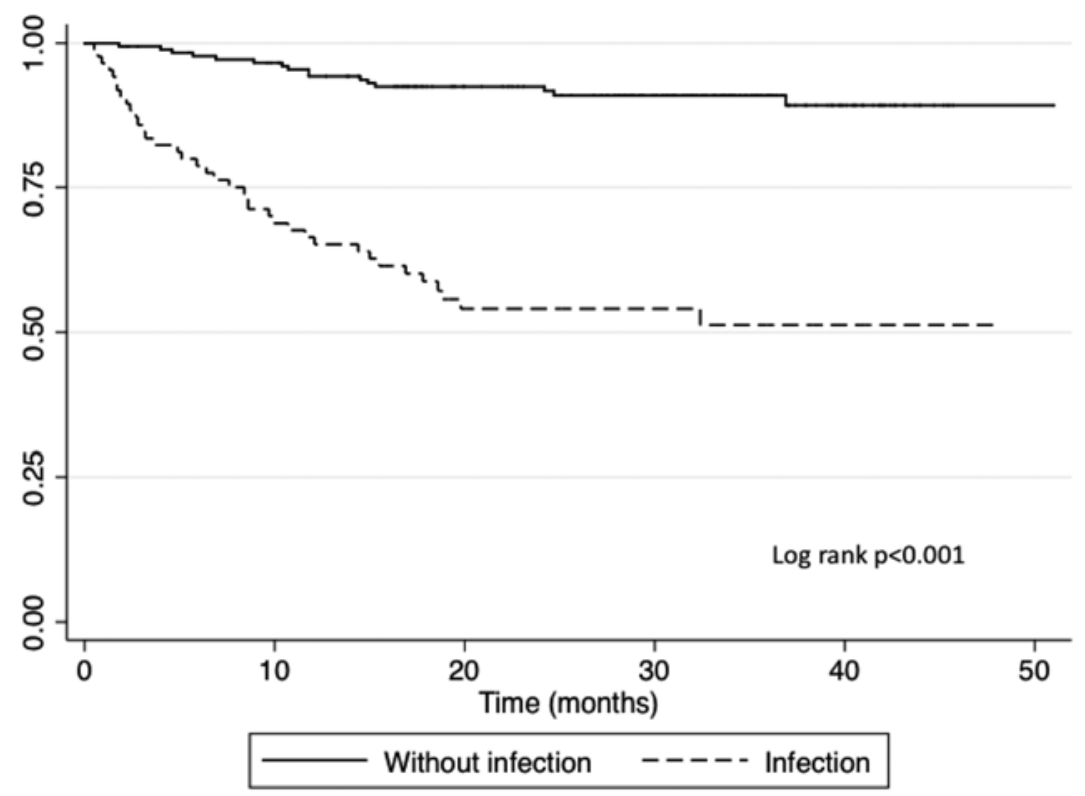

developed mycobacterial infection had a diagnosis of DM. The high incidence rate reported in our study, in the first 2 years of receiving rituximab, warrants the recommendation to investigate $\mathrm{LTBI}$ and to prescribe prophylaxis in patients expected to be treated with rituximab.

There were not patients with HBV reactivation. Although we did not perform HBV-DNA in all patients, none had $\mathrm{HBsAg}$ positive. In those patients who presented transaminases elevation, HBV serology was repeated, and no patient had $\mathrm{HBsAg}$ seroconversion.

We did not include patients with HIV in this study; these individuals comprise a special group with a higher risk for infections (around $12 \%$ ), particularly opportunistic pathogens in patients with CD4 of fewer than 50 cells $/ \mathrm{mm}^{39,14}$.

There were more patients with an infection that did not achieve complete tumor response with the first chemotherapy scheme when compared with those without infections $(p<0.0001)$. This could be related to a delay to receive timely cycles or a decrease in chemotherapy dosing.

Eighteen patients presented with some neoplasm: 13 asynchronous and 5 synchronic. During the follow-up, no patient developed a new neoplasm, which was probably related to the short median follow-up time (19 months).

Likewise, infectious processes, along with age above 60 years, and failure to achieve $C R$ were risk factors associated with mortality. A study that included 325 patients with diffuse large B-cell lymphoma who received rituximab and who was followed for 10 years, the incidence of infection was $63.4 \%$. Infection was an independent predictor of death, in addition to age, the Charlson Comorbidity Index, Eastern Cooperative Oncology Group performance status, and neutropenia $^{15}$. In the past years, several infectious agents have been reported as involved in the malignant transformation of B or T lymphocytes, and therefore associated with the pathogenesis of Iymphoproliferative disorders ${ }^{16}$. Furthermore, the antigenic infectious stimulation was shown to be fundamental in the cell perturbation of the microenvironment in sustaining the neoplastic cell growth, which can condition failure in response to oncology treatment ${ }^{16}$. Although this hypothesis is interesting, this study does not intend to establish that infections stimulate or condition a favorable environment for tumor growth.

Study weaknesses include that data derived from a single oncology center; therefore, these data may not 
apply in the same way to other populations, in that local epidemiology and local policies may vary. PPD was not performed in all patients, so no timely diagnosis of LTBI was made, and therefore prophylactic treatment was not prescribed before the start of rituximab. All the patients had a baseline serological study for hepatitis; however, this was not repeated continuously either semi-annually or annually to assess if seroconversion occurred; it was only performed in those with elevated transaminases to rule out infection or reactivation of viral hepatitis. Rituximab doses can be different between different lymphoma subtypes; however, the cumulative doses have a long-term effect. Lack of molecular testing for some pathogens is one of the main problems that the majority of government hospitals face in middle and low income countries.

The study's strengths are the inclusion solely of patients with lymphoma; thus, we had a captive population that allowed us to follow them. To emphasize the importance of conducting screening tests for TB in all patients who will receive rituximab. Moreover, a continuous follow-up even when they have finished the chemotherapy scheme for at least 2 years.

The most important finding was the development of infectious complications in patients with $\mathrm{NHL}$ receiving first-line chemotherapy with R-CHOP which increased the risk of mortality.

\section{ACKNOWLEDGMENTS}

We thank Dr. Ana Florencia Ramírez-Ibarguen for her support.

\section{REFERENCES}

1. Lanini S, Molloy AC, Fine PE, Prentice AG, Ippolito G, Kibbler CC, et al. Risk of infection in patients with lymphoma receiving ritux- imab: systematic review and meta-analysis. BMC Med. 2011;9:36

2. Gea-Banacloche JC. Rituximab-associated infections. Semin Hematol. 2010;47:187-98.

3. CTCAE Common Terminology Criteria for Adverse Events version 4.02. National Institute of Health. National Cancer Institute; 2009. Available from: https://www.evs.nci.nih.gov/ftp1/ CTCAE/Archive/CTCAE 4.02 2009-09-15 QuickReference_8.5x11.pdf. [Last accessed on 2016 Feb 03].

4. See I, Iwamoto $\bar{M}$, Allen-Bridson K, Horan T, Magill SS, Thompson ND, et al. Mucosal barrier injury laboratory-confirmed bloodstream infection: results from a field test of a new national healthcare safety network definition. Infect Control Hosp Epidemiol. 2013:34:769-76.

5. National Health Safety Network (NHSN) Surveillance Definitions: Central-Line Associated Bloodstream Infection (CLABSI) and Related Sites. Final 06 2006. Available from: http://www. msic-online.org/pdf/NHSN_Definitions_CLABSI.pdf. [Last accessed April 1, 2017]

6. Tudesq JJ, Cartron G, Rivière S, Morquin D, lordache L, Mahr A, et al. Clinical and microbiological characteristics of the infections in patients treated with rituximab for autoimmune and/or malignant hematological disorders. Autoimmun Rev. 2018; 17:115-24

7. Alonso JJ, Cánovas A, Barreiro JG, Aguirre C. Infectious complications of chemotherapy in clinically aggressive mature B and T cell lymphomas. Eur J Intern Med. 2012;23:255-60.

8. Kasi PM, Tawbi HA, Oddis CV, Kulkarni HS. Clinical review: serious adverse events associated with the use of rituximab-a critical care perspective. Crit Care. 2012;16:231.

9. Kelesidis T, Daikos G, Boumpas D, Tsiodras S. Does rituximab increase the incidence of infectious complications? A narrative review. Int J Infect Dis. 2011;15:e2-16.

10. Carson KR, Focosi D, Major EO, Petrini M, Richey EA, West DP et al. Monoclonal antibody-associated progressive multifocal leucoencephalopathy in patients treated with rituximab, natalizumab, and efalizumab: a review from the research on adverse drug events and reports (RADAR) project. Lancet Oncol. 2009; 10:816-24.

11. Souto A, Maneiro JR, Salgado E, Carmona L, Gomez-Reino JJ. Risk of tuberculosis in patients with chronic immune-mediated inflammatory diseases treated with biologics and tofacitinib: a systematic review and meta-analysis of randomized controlled trials and long-term extension studies. Rheumatology (Oxford). 2014;53:1872-85

12. World Health Organization. Global Health Observatory Data Repository. Incidence Data by WHO Region. Number of Incident Tuberculosis Cases. Updated 07 Oct 2017. Available from: ht t p : / / w w.apps.who.int / gho/data / view. main.57036ALL?lang=en. [Last accessed on 2017 Nov 10].

13. Castellanos-Joya M, Delgado-Sánchez G, Ferreyra-Reyes L, Cruz-Hervert P, Ferreira-Guerrero E, Ortiz-Solís G, et al. Results of the implementation of a pilot model for the bidirectional screening and joint management of patients with pulmonary tuberculosis and diabetes mellitus in mexico. PLoS One. 2014; 9:e106961.

14. Zallio F, Limberti G, Ladetto M. Infections and follicular lymphoma: is there a link? Mediterr J Hematol Infect Dis. 2017; 9:e2017035

15. Dendle C, Gilbertson M, Spelman T, Stuart RL, Korman TM, Thursky K, et al. Infection is an independent predictor of death in diffuse large B cell lymphoma. Sci Rep. 2017;7:4395.

16. Lanini S, Molloy AC, Prentice AG, Ippolito G, Kibbler CC. Infections in patients taking rituximab for hematologic malignancies: two-year cohort study. BMC Infect Dis. 2013;13:317. 